\title{
An analysis of the utilization review process in the Illinois workers' compensation system
}

\begin{abstract}
Background: Utilization review (UR) has increasingly been utilized in the Illinois workers' compensation system to review the appropriateness of recommended treatment by the treating physician. As the UR process has become more prevalent, the ability of the physician to recommend appropriate treatment has become more burdensome.

Methods: We performed a case series $(\mathrm{N}=42)$ of utilization reviews sent to an orthopedic surgeon's (author BAR) office from April 2018 until January 2020. All UR submissions were analyzed for denial timelines and reasoning. The surgeon responded to all denials in writing in a timely fashion and based upon the belief the treatment was reasonably required to cure or relieve the effects of his or her injury.

Results: Of the 42 utilization reviews, 40 (95\%) were denied throughout the process and never approved. One treatment was approved, and the treating surgeon agreed with one UR denial. Even though almost all treatment recommendations never received formal approval, $46 \%$ (17 of 37 ) of the treatments received payment for the service. Only 33\% (14 of 42) of the physician UR responses were paid.

Conclusion: We found that only one recommended treatment $(\mathrm{N}=42)$ received authorization. On average, the insurer issued 4.3 UR denials per patient and denied payment $54 \%$ of the time for the recommended treatment. The process of utilization review is being implemented as a method to control costs by denying treatment based upon suspect clinical practice guidelines.
\end{abstract}

Keywords: utilization review, worker's comp, evidence based medicine, clinical practice guidelines, official disability guidelines, Illinois worker's compensation, Illinois workers' compensation act
Volume 12 Issue 3 - 2020

\author{
Blair A Rhode, ' William S Rhode² \\ 'Orthopedic Surgeon, Orland Park Orthopedics, USA \\ ${ }^{2}$ Rhode Orthopedic Research Institute (RORI), USA
}

Correspondence: Blair A Rhode, Orland Park Orthopedics, I 6450 S 104th AV, Orland Park, Illinois 60467, USA, Tel 708-364-844I, Fax 708-364-8443.

Email Blairbons@gmail.com

Received: May 20, 2020 | Published: June 03, 2020
Abbreviations: UR, utilization review; ODG, official disability guidelines; EBM, evidence based medicine; CPGs, clinical practice guidelines; StDev, standard deviation

\section{Utilization review}

A critical evaluation (as by a physician or nurse) of health-care services provided to patients that is made especially for the purpose of controlling costs and monitoring quality of care. - Merriam-Webster.

\section{Introduction}

Utilization review (UR) was originally introduced in the 1960's with the intent to reduce overutilization and eliminate waste. Review was traditionally performed by registered nurses in the hospital setting but gained popularity within the insurance industry as research was published in the areas of medical necessity, misuse, and overutilization of services. There are three activities within the utilization review process: prospective, concurrent and retrospective. Prospective review includes the review of medical necessity for the performance of services or scheduled procedures before admission. Concurrent reviews include a review of medical necessity decisions made while the patient is currently in an acute or post-acute setting. Retrospective reviews involve a review of coverage after treatment is provided. ${ }^{1}$

The utilization review process has increasingly been applied to the treatment of workers injured on the jobsite. Workers' Compensation is a state mandated no-fault system form of insurance that ensured that workers injured at work would receive compensation without delay and without regard to fault. It was started in response to serious societal problems caused by a dramatic rise in the number of people injured in industrial settings. It is a benefit provided in exchange for mandatory relinquishment of the employee's right to sue his or her employer under the common civil law of negligence.

Employers and insurance companies have increasingly complained that workers' compensation costs are making states less competitive even though employers are paying the lowest rate for workers' compensation than at any time in the last 25 years (Table 1). From 1991 to 2014 , the cost of worker's compensation insurance has decreased $26 \%$ while the cost of health insurance has increased $46 \%{ }^{2}$

Table I Cost to employers for $\$ 100$ of workers' wages ${ }^{3}$

\begin{tabular}{lll}
\hline & 1991 & $\mathbf{2 0 1 4}$ \\
\hline Cost of health insurance & $\$ 8.55$ & $\$ 12.52$ \\
Cost of retirement and savings & $\$ 5.50$ & $\$ 7.29$ \\
Cost of workers' comp & $\$ 2.71$ & $\$ 2.00$ \\
\hline
\end{tabular}

The state of Illinois enacted a medical fee schedule in 2006 and implemented further reductions in 2011 with the intent to decrease the workers' compensation costs. These cuts resulted in insurers reporting a 19\% decrease in total benefit payments from 2011-2015 (Chairman 
of the Workers' Compensation Committee Joan Fratianni letter to the governor dated June 16, 2015). The Illinois Worker's Compensation act from 2011 furthermore codified the utilization review process such that treating physicians are required to submit to the process.

The Illinois Worker's Compensation act section 8.7 states that upon receipt of written notice that the employer or the employer's agent or insurer wishes to invoke the utilization review process, the provider of medical, surgical, or hospital services shall submit to the utilization review, following accredited procedural guidelines. In fact, an employer may deny payment of or refuse to authorize payment of medical services rendered or proposed to be rendered on the grounds that the extent and scope of medical treatment is excessive and unnecessary in compliance with an accredited utilization review program under this Section.

When a payment for medical services has been denied or not authorized by an employer or when authorization for medical services is denied pursuant to utilization review, the employee has the burden of proof to show by a preponderance of the evidence that a variance from the standards of care used by the person or entity performing the utilization review pursuant to subsection (a) is reasonably required to cure or relieve the effects of his or her injury. An admissible utilization review shall be considered by the Commission, along with all other evidence and in the same manner as all other evidence, and must be addressed along with all other evidence in the determination of the reasonableness and necessity of the medical bills or treatment. ${ }^{4}$

The insurance industry often utilizes clinical practice guidelines such as the ones sold by the Work Loss Institute as part of their utilization review process to determine the appropriateness of particular treatment regimens. Most clinical practice guidelines promote the fact that they are closely aligned with evidence base medicine. Evidencebased medicine (EBM) has been defined as the conscientious, explicit and judicious use of the best evidence for making the best decisions for patient care. Strict adherence to some set of established guidelines places the physician at risk for malpractice suits, as the standard may not be in the best interest of the individual patient.

The results of some research studies are statistically weak or incorrect and have never been replicated by other investigators. It has been stated, "Most published research findings are false". An extensive review by Ioannidis and colleagues has repeatedly documented the shortcomings of clinical studies. Bias occurs as a result of design, data, analysis and presentation factors that produce findings when they shouldn't. Even when research claims are strongly contradicted by the results of randomized trials, they have been shown to persist in the literature. ${ }^{6}$ Given the time lag for development of the Clinical Practice Guidelines (CPGs), they may be out of date in part or whole due to the rapidly expanding medical knowledge base. ${ }^{7}$ Only 50 percent of CPGs were found to be valid after 5.8 years.

We have found that the utilization review process has been utilized as a method to create barriers to access to appropriate medical care. Rather than engage in a process where the UR physician considers all evidence in determining treatment appropriateness, clinical practice guidelines are being weaponized as a method of denial by declaring them dispositive. We have recently performed an analysis of a consecutive series of utilization reviews to determine denial/approval patterns and their effect on quality and access to care.

Methods: We performed a case series $(\mathrm{N}=42)$ of utilization reviews sent to an orthopedic surgeon's (author BAR) office from April 2018 until January 2020. Treatment recommendations included surgery, physical therapy, oral medications, topical medications, diagnostic tests and post-operative cold therapy. Every time the UR institution sent a denial, the treating surgeon formulated a written response that adhered to the requirements of the Illinois Workers' Compensation Act. Specifically, the physician's response documented why the treatment was reasonably required to cure or relieve the effects of his or her injury. If the treating physician agreed with the UR denial, the recommended treatment discontinued. If the treating physician felt the treatment was reasonable and necessary, he complied with the Act's requirements of responding to the denial. Responses justified why the recommended treatment was appropriate to cure or alleviate the injured workers' condition. Responses also analyzed the appropriateness of the clinical practice guidelines and also reviewed other factors such as relevant literature not referenced by the guidelines.

The initial treatment date, utilization review date, UR response date, and last visit date were recorded. The type of UR review was documented (Retrospective, prospective, concurrent). The clinical practice guideline utilized for the review was recorded. The first and last UR date was recorded; approval status and number of utilization reviews were also documented. Payment status for the treatment and the physician UR response were also recorded. Utilization reviews were counted based upon recurrent responses for the particular treatment recommendation included in the study (UR) and total utilization reviews (total UR).

\section{Results}

Of the 42 utilization reviews, 40 (95\%) were denied throughout the process and never approved (Table 2). One treatment was approved by the UR physician. The treating surgeon agreed with one UR denial and treatment was subsequently discontinued. Even though almost all treatment recommendations never received formal approval, 46\% (17 of 37) of the treatments received payment for the service. Only $33 \%$ (14 of 42) of the physician UR responses were paid. There was an average of 4.3 reviews performed per patient with a range of 1 to 16 unique utilization reviews (Table 3). 40 of the 42 reviews (95\%) cited the Official Disability Guidelines (ODG) as the clinical practice guideline utilized for reference. Only4 of $42(9.5 \%)$ of the reviews were prospective while $90.4 \%$ (38 of 42) were retrospective reviews. No utilization reviews were concurrent.

Table 2 UR and payment denial rates

\begin{tabular}{ll}
\hline Action & Rate \\
\hline UR Denial & $95 \%$ \\
Treatment payment denial & $54 \%$ \\
Theater UR payment denial & $67 \%$
\end{tabular}

Table 3 Utilization review totals

\begin{tabular}{llll}
\hline Statistic & URs & Total URs & $\begin{array}{l}\text { Total duration for } \\
\text { denial (days) }\end{array}$ \\
\hline count & 89 & 181 & 40 \\
average & 2.12 & 4.31 & 209.2 \\
StDev & 1.27 & 3.35 & 136.1 \\
minimum & I & 1 & 29 \\
maximum & 5 & 16 & 633 \\
\hline
\end{tabular}




\section{Discussion}

The denial rates shown in Table 2 demonstrate that when the treating physician is requested to engage in the utilization review process, the likelihood of achieving a written approval for the treatment is extremely unlikely. Even if the theater complies with all components of the Illinois Worker's Compensation act, there is a 95\% chance that the UR physician will deny treatment. Given the fact that over $90.4 \%$ of these reviews was retrospective, the intent of the UR was to deny payment for services that were already rendered.

Under the Illinois Workers' Compensation Act Section 8.7(i), the conclusion rendered by the UR physician is not intended to be dispositive. It is intended to be "considered by the Commission, along with all other evidence and in the same manner as all evidence, in the determination of the reasonableness and necessity of the medical bills or treatment." Hardy v. Murray Developmental Center, 07 WC 48727, 09 I.W.C.C. 0725, 2009 WL 2516197 (July 15, 2009). The injured worker has the burden of proof to show that the recommended medical treatment under question (variance from the standards of care used by the person or entity performing the utilization review) is reasonably required to cure or relieve the effects of his or her injury.

We found that $95 \%$ of the UR denials used the Official Disability Guidelines (Work Loss Institute) as their clinical practice guidelines reference. The Work Loss Advisor Board was previously analyzed and found to have 103 members. The guidelines encompass a wide variety of medical conditions and as such the members included at least 9 chiropractors, 12 psychologists \& pain physicians, at least 20 stakeholders from insurance companies and employers. There were only 10 orthopedic surgeons listed of which 4 were Emeritus (retired), 3 were spine specialists and others were in medical business or management. ${ }^{8}$ Furthermore, the Guidelines do not reference the actual advisor or their qualifications that authored each the ODG subsection. The Guidelines also does not allow access to the proprietary patient data subsets for critical review. Their recommendations were often found to be an editorial conclusion rather that an unbiased assessment of the available data.

An example was the ODG recommendation for physical therapy for rotator cuff pathology. The Official Disability Guidelines (ODG) PT Guidelines for complete rupture of the rotator cuff recommends 24 visits for a standard arthroscopic rotator cuff repair. ${ }^{9}$ Even though the guidelines note that their treatment planning section is not designed to be a rule and as such not to be used as a basis for Utilization Review, we found that the UR physicians did indeed use this section as a hard recommendation.

Review of the ODG subsection for physical therapy after rotator cuff repair demonstrates that only two articles were referenced as support for the guidelines. ${ }^{10}$ The Ejnisman et al. ${ }^{10}$ article looked at the evidence for the superiority of open rotator cuff repair versus arthroscopic debridement. The only reference to physical therapy was directed at physical therapy versus placebo in the non-operative patient. The second article by Byram et al. ${ }^{11}$ assessed the correlation between preseason screening for prone external rotation strength loss and in season injury and contained no analysis of post-operative physical therapy. ${ }^{11}$ Neither of these articles assessed physical therapy after surgical repair of a rotator cuff in any manner. The only study that has analyzed physical therapy utilization in arthroscopic rotator cuff repairs performed on workers' compensation patients demonstrated an average of 43 visits $(\mathrm{SD}=13.7){ }^{8}$ Based upon the only study that looked at post-operative therapy in the workers' compensation rotator cuff patient, 29 to 57 (Average $=43$ ) physical therapy visits would be appropriate.

Another treatment recommendation that was often referred for utilization review was the use of the oral medication cyclobenzaprine. The most common basis for denial was that this medication is only indicated for short term use up to 14 days. Review of the ODG citation declares that "treatment should be brief; this medication is not recommended for longer than 2-3 weeks". ${ }^{9}$ The recommendation was based upon an article performed by Browning et al. ${ }^{12}$ directed at the use of this drug for treatment of back pain. Review of the article demonstrates the author actually concluded that that it is impossible to say what the optimum duration of treatment is. In the first 4 days of treatment, patients experienced moderate degrees of improvement. Although the optimum duration of treatment may be short, they concluded that it was unclear as to the optimum duration of treatment was, since even at 14 days there was evidence of improvement with treatment. Furthermore, extrapolation of this data directed at the treatment of back pain and other conditions was not explained.

\section{Conclusion}

Utilization review has gained in popularity in its use to lower treatment costs in the workers' compensation environment. Even though it is a state mandated no-fault system of insurance that is intended to provide the injured worker compensation and treatment without delay and without regard to fault, we have found systems have been put in place that has limited appropriate access to treatment. We have previously shown that denying causation of injury can increase time to maximum medical improvement (MMI) from 5.7 months to 47 months with an increase of $\$ 200,000$ in treatment costs. ${ }^{13}$ We have also demonstrated that forcing patients to use outside pharmacies from the doctor's office significantly limits the patient's access to medicines that are covered by the workers' compensation insurer. Only one patient in the study was dispensed the prescribed medication from the physician with none of the patients being dispensed their medication at their index visit to the pharmacy. ${ }^{14}$ This current study demonstrates that the UR process is being weaponized as a method to deny approval and ultimately payment for recommended treatment.

We found that only one recommended treatment $(\mathrm{N}=42)$ received authorization. Patients received 4.3 UR denials with one patient receiving 16 unique UR denials. All of the UR denials underwent a response by a double board certified, orthopedic surgeon that followed the ODG guidelines and pertinent literature. Furthermore, it was the position of the treating physician that all treatment was reasonably required to cure or relieve the effects of his or her injury. Ultimately, this is the standard that the treating physician must meet to find a treatment necessary. Unfortunately, we found that the insurer intended the utilization review process to be dispositive and not to be treated as evidence to be assessed like all other evidence.

We found that most repeat UR responses did not respond to the surgeon's response. They continued to use the same clinical practice guideline referenced in the prior denial without addressing the surgeon's opinion. We conclude that the UR process is being utilized to create barriers to care and not create rational dialog.

\section{Acknowledgments}

None. 


\section{Conflicts of interest}

The authors declare there are no conflicts of interest.

\section{Funding}

None.

\section{References}

1. https://www.mcg.com/blog/2018/06/21/utilization-review-medicalnecessity/

2. Bureau of Labor Statistics National Compensation Survey 1991 - 2014.

3. Rhode BA. The three D's of insurance: delay, deny and do not payanalysis of collection rates for illinois workers' compensation patients. MOJ OrthopRheumatol. 2015;3(1):267-269.

4. Illinois Worker's Compensation act 820 ILCS 305/8.7.

5. Ioannidis J. Why most published research findings are false. PLoS Medicine. 2005;2(8):696-701.

6. Tatsioni A, Boitsis NG, Ioannidis JP. Persistence of contradicted claims in the literature. JAMA. 2007;298(21):2517-2526.
7. Shekelle PG, Ortiz E, Rhodes S, et al. Validity of the Agency for Healthcare Research and Quality Clinical Practice Guidelines: How quickly do guidelines become outdated. JAMA. 2001; 286(12):1461-1467.

8. Rhode B. Utilization analysis of post-operative physical therapy for rotator cuff repair (2017). MOJ OrthopRheumatol. 2018;10(3):184-187.

9. Official Disability Guidelines ${ }^{\circledR}\left(23\right.$ rd annual edition) \& ODG ${ }^{\circledR}$ Treatment in Workers' Comp (16th annual edition). See Top 6 chapters. Shoulder.

10. Ejnisman B, Andreoli CV, Soares BG, et al. Interventions for tears of the rotator cuff in adults. Cochrane Database Syst Rev. 2004;(1):CD002758.

11. Byram IR, Bushnell BD, Dugger K, et al. Preseason shoulder strength measurements in professional baseball pitchers: identifying players at risk for injury. Am J Sports Med. 2010;38(7):1375-1382.

12. Browning R, Jackson JL, O’Malley PG. Cyclobenzaprine and back pain: a meta-analysis. Arch Intern Med. 2001;161(13):1613-1620.

13. Rhode BA, Rhode WS, Rhode BJ. Removal of Barriers to Care Result in Improved Outcomes for Manual Laborers with Compressive Neuropathies. MOJ Orthop Rheumatol. 2015;2(2):62-65.

14. Rhode BA, Rhode WS. In office pharmacy and its relation to access to care in workers' compensation. MOJ Orthop Rheumatol. 2018;10(3):172-173. 